\title{
Nordiques
}

$34 \mid 2017$

La mer Baltique comme zone-frontière : perspectives environnementales, géopolitiques, culturelles

\section{Les États baltes vus de Moscou}

Jean-Christophe Romer

\section{(2) OpenEdition}

Journals

Édition électronique

URL : https://journals.openedition.org/nordiques/1754

DOI : $10.4000 /$ nordiques. 1754

ISSN : $2777-8479$

Éditeur :

Association Norden, Bibliothèque de Caen la mer

Édition imprimée

Date de publication : 1 novembre 2017

Pagination : $99-112$

ISBN : 9791095914006

ISSN : 1761-7677

Référence électronique

Jean-Christophe Romer, « Les États baltes vus de Moscou », Nordiques [En ligne], 34 | 2017, mis en

ligne le 21 octobre 2021, consulté le 27 novembre 2021. URL : http://journals.openedition.org/

nordiques/1754; DOI : https://doi.org/10.4000/nordiques.1754 


\section{Les États baltes vus de Moscou}

Jean-Christophe Romer*

\section{RÉSUMÉ}

Anciens territoires de l'Empire russe, puis républiques de l'Union soviétique, les trois États baltes ont vu leur situation instrumentalisée par Moscou dès la disparition de l'URSS. Entre volonté centrifuge de ces derniers et vision impériale de la Russie, les situations de crise, voire de confrontation, étaient presque inévitables. À des degrés divers, la confrontation s'exprime dans plusieurs domaines, de la représentation de l'espace à celle de la sécurité en passant par des rivalités d'écriture d'histoire et de mémoire. Ces trois États constituent dès lors pour la Russie un lieu privilégié et même un fer de lance de l'expression des rivalités de pouvoir entre Moscou et le monde occidental.

ABSTRACT

Since the re-acquisition of their independence at the end of the Cold War, the Baltic states have been used by Russia in its international relations. Their will to strongly separate themselves from their Soviet past has clashed directly with the Russian imperial nostalgia, and crisis situations have inevitably arisen. This confrontation is expressed at various levels and in various ways, from strategic representation of the Baltic area to security concerns and quarrels about memories and historical narratives. These states have thus provided contemporary Russia with a focal point for the expression of its rivalry with the Western world.

\footnotetext{
* Jean-Christophe Romer, professeur, enseigne à l'Institut d'études politiques de Strasbourg.
} 


\section{INTRODUCTION}

Les États baltes ont, depuis le XVIII e siècle, constitué pour la Russie à la fois une partie intégrante de leur Empire, qu'il ait été tsariste ou soviétique, et un espace quelque peu marginal au sein de ce même Empire ; partie intégrante, car ces "provinces", comme on les désignait du temps des tsars, ont donné à la Russie nombre de grands responsables politiques ou militaires - Barclay de Tolly ou Witte pour n'en citer que deux -, mais aussi partie marginale pour des questions culturelles. Ces territoires ne sont ni slaves ni orthodoxes, leur élites sont allemandes et ils furent souvent économiquement plus avancés que le reste de l'Empire. Pour ne parler que du XX $X^{e}$ siècle, cela a conduit à l'écriture d'une histoire contrastée, depuis le régiment des tirailleurs lettons ralliés aux bolcheviks en 1917 jusqu'à la légion lettonne intégrée à la Waffen SS. Aujourd'hui les Lettons commémorent volontiers les seconds, oubliant totalement les premiers pour la plus grande indignation des Russes.

Les trois États baltes sont aussi, parmi les Républiques ex-soviétiques, les seuls à avoir accédé à une indépendance véritable et durable et à avoir fait fonctionner normalement des institutions (plus ou moins) démocratiques, une génération durant entre 1920 et 1940. Aucune autre République soviétique, Russie comprise, n'a connu une telle expérience. Enfin, ceci expliquant en partie cela, ce sont les toutes premières Républiques à avoir proclamé leur souveraineté (entre novembre 1988 et juillet 1989) puis, entre mars et mai 1990, annoncé des indépendances ${ }^{1}$ dont l'effet sera suspendu à la demande de la France et de l'Allemagne jusqu'en août 1991.

Enfin, leur localisation dans l'espace européen constitue aussi un facteur explicatif des relations complexes que la Russie entretient avec ces trois États. Marches de l'Empire russe, les trois Baltes sont tout autant les marches d'un hypothétique "Empire » européen, voire euro-atlantique. On ne peut pas pour autant les assimiler à la Biélorussie ou à l'Ukraine en ce qu'ils ont une histoire et une culture qui leur ont permis d'exprimer plus clairement leur identité et leur volonté d'indépendance que les autres Républiques européennes de l'ex-URSS. De plus, même si les trois pays baltes se sont, depuis leur adhésion à l'OTAN, souvent rapprochés de la Pologne, ils n'en ont pas moins une différence avec celle-ci, essentielle aux yeux de Moscou : les uns faisaient partie de l'Union soviétique, l'autre non. Contrairement à ce que, malheureusement, l'on peut parfois lire ou entendre sous l'effet d'un raccourci tout aussi simplificateur que faux, les Baltes n'ont jamais été membres du pacte de Varsovie : de 1945 à 1991, ils n'étaient pas des États souverains, cette souveraineté fût-elle limitée.

1 Le 11 mars 1990 pour la Lituanie, le 30 mars pour la Lettonie et le 4 mai pour l'Estonie. 
La volonté centrifuge des trois Baltes s'est toutefois exprimée parfois de manière sinon violente tout au moins provocatrice, ce à quoi la russe a évidemment répondu de façon tout aussi provocante. Cela se manifeste notamment dans des lectures très personnelles d'une histoire commune perçue de manière très différente de part et d'autre. En un mot, les relations entre la Russie et ses "provinces perdues » se sont essentiellement articulées autour de deux grandes questions récurrentes : une guerre des histoires et des mémoires, étroitement liée à la question des minorités russes dans ces trois États. Le second grand contentieux concerne les questions stratégiques et diplomatiques et, notamment, celle de la sécurité aux frontières après l'adhésion des trois États à l'Union européenne et surtout à l'OTAN. Mais tout cela s'inscrit dans une problématique d'identification de la région.

\section{L'IDENTIFICATION D’UNE RÉGION}

La question de la localisation des trois États baltes dans l'espace européen n'est pas récente : elle a ressurgi à plusieurs moments de l'histoire, dont naturellement après 1991. Ces États sont-ils partie de l'Europe centrale - ce qui serait surtout pertinent pour la Lituanie -, de l'Europe du Nord ou de celle de l'Est, au même titre que la Biélorussie ou l'Ukraine ? Il est clair que ces trois États se situent d'abord à un carrefour d'espaces culturel, c'est-à-dire, a priori, sur des lieux de lutte d'influence'.

Si ces questions ont pu se poser surtout au moment des processus de dislocation de l'Empire soviétique et de ce que M. Gorbatchev avait qualifié de "parade des souverainetés " amorcée en 1988, elles restent au cœur des réflexions de nombre d'universitaires russes. Certes, il convient de replacer ces questions à leur juste mesure afin de ne pas surestimer la place que les trois États baltes occupent dans la recherche en sciences sociales en Russie. Si l'on observe le poids qu'occupent ces trois pays, et la région baltique dans son ensemble, dans les travaux de l'Institut d'Europe, le principal institut de l'Académie des sciences censé traiter de ces questions, l'on ne peut que constater la faible importance quantitative des publications consacrées à cette région. Or cet institut est plutôt orienté vers des logiques occidentalistes comme l'avait noté son ancien directeur, Nikolaï Chmelev dans le manuel de référence de l'Institut : "La Russie a été et sera toujours d'Europe. " ${ }^{3}$ Il est vrai que l'ouvrage a été publié en 2002, c'est-à-dire en des temps plus « europhiles».

2 Jean-Christophe Romer, "Les États baltes entre Nord, Centre et Est ", Stratégique, no 55, 1992, p. $97-114$.

3 Evropa vtchera, segodnja, zavtra (Europe, hier, aujourd'hui, demain), N. Chmelev (dir.), Moscou, Ekonomika, 2002. 
Dans ce même manuel, la partie consacrée aux questions du régionalisme européen traite notamment de l'Europe du Nord et de la Baltique et aborde la question de sa localisation. Pour les auteurs de ce sous-chapitre, l'intégration des trois États baltes après 1991 permet de former la "nouvelle Europe du Nord » comprenant les cinq États traditionnels auxquels « s'ajoutent les États baltes et les régions nord-ouest de la Russie, y compris les territoires riverains de la mer de Barents jusqu’à la région dite "euro-arctique" ". À cet ensemble nouveau, il convient d'inclure l'Allemagne et la Pologne $e^{4}$. La Baltique occupe donc une place honorable dans cet ouvrage de référence qui semble d'abord insister sur une volonté d'intégration de la Russie dans un nouvel ensemble régional. D'ailleurs, Moscou avait manifesté son souhait d'être intégrée dans le cadre d'un éphémère projet de l'UE sur la "dimension septentrionale ", très à la mode dans le début de la décennie 2000 mais quelque peu oublié aujourd'hui, sauf peut-être par la Russie elle-même . Il est vrai que le début de cette décennie est marqué par une réelle volonté de rapprochement avec l'Europe, y compris au plus haut niveau de l'État.

À l'inverse, il est tout aussi intéressant de constater que dans un autre ouvrage publié par l'Institut d'Europe en 2008, sous la direction de deux des auteurs de l'article mentionné ci-dessus, et consacré à l'Europe du Nord, il n'est question que des "cinq États traditionnels", y compris lorsqu'il est question de l'adhésion à l'OTAN ou à l'UE. Les trois États baltes sont ainsi mentionnés une seule fois dans l'ouvrage, dans la liste de pays participant au Conseil des États de la Baltique. L'absence de ces États n'est d'ailleurs pas justifiée dans l'introduction de l'ouvrage ${ }^{6}$. Cette faible présence se manifeste aussi dans les grands textes programmatiques russes tels que la doctrine militaire ou la stratégie de sécurité nationale, dont les dernières « éditions " datent respectivement du 26 décembre 2014 et du 31 décembre 2015.

Une possible explication de ce (faux) manque d'intérêt pour cette région peut tenir au fait que les trois États qui la composent sont parfois considérés comme de "petits États » ressortissant à une théorie du même nom qui insiste sur plusieurs points conduisant la Russie à ne pas nécessairement lui porter d'intérêt direct mais à les considérer toujours en « relation à ». Les petits États - baltes, en l'occurrence ne sont en effet pas à même d'assurer seuls leur sécurité, se développent dans un système international décentralisé et privilégient des modèles asymétriques

4 Natalia Antjuchina, Juri Derjabin, Evgenii Kutovoj, «Sever Evropy i Baltija. Severnoe izmmerenie » («L'Europe du Nord et la Baltique. La dimension septentrionale»), in N. Chmelev (dir.), op. cit., p. 434-435. Les cinq États «traditionnels » sont le Danemark, la Finlande, l'Islande, la Norvège et la Suède.

5 Voir notamment : Concept de politique étrangère de la Fédération de Russie, Ukaz (ordre) du président du 30 novembre 2016, (\$ 71), [http://www.kremlin.ru/acts/bank/41451] (consulté le 15 septembre 2017).

6 Severnaja Evropa region novogo razvitja (L'Europe du Nord, une région de développement nouveau), N. Antjuchina et J. Derjabin (dir.), Moscou, Ves' Mir [Institut d'Europe], 2008. 
de relations interétatiques ${ }^{7}$. Ces États sont ainsi vus comme étant caractérisés par une propension à l'internationalisation des risques et par leur préférence pour une moralisation normative de la politique mondiale ${ }^{8}$. En un mot, de tels pays privilégient un modèle conflictuel de relations, se refusent à tout compromis et cherchent à sécuriser leur politique en institutionnalisant leur intégration à l'ouest (adhésion à l'UE et à l'OTAN). Cet ensemble de caractéristiques conduirait alors à une montée des nationalismes par le biais notamment d'une politisation du facteur ethnique'. Si l'auteur de l'article ne va pas jusqu'à l'écrire, ce qui est sous-jacent à son propos est finalement que les petits États (baltes) constituent bien des sources de conflit, des perturbateurs, car « l'asymétrie donne souvent la supériorité au plus faible $»^{10}$. Par contre, il n'y est pas question d'un quelconque rôle positif tel celui de médiateur que les « petits États » ont pu exercer dans l'histoire et qui leur est fréquemment attribué.

Cette vision toute théorique des petit États trouve naturellement une claire application dans les deux principaux éléments qui jalonnent les relations entre la Russie et ses voisins baltes : la question ethno-historique et celle des garanties de sécurité.

\section{HisTOIRE, MÉMOIRE, MINORITÉS}

Tous les États issus de l'URSS ont procédé à des réécritures de leur histoire nationale. Les États baltes n'ont pas fait exception à la règle, une légende se substituant à une autre. Ils n'ont pas suivi le conseil d'Adam Michnik lorsque, face au phénomène de "lustration ", il mettait en garde de ne pas écrire une nouvelle histoire "officielle" se substituant à la précédente ${ }^{11}$. Ainsi, l'idée majeure que veulent faire passer les Baltes est celle d'une histoire plus que millénaire au cours de laquelle ils n'auraient jamais été dominés par quiconque mais seulement " occupés " par l'URSS de 1940 (1945) à 1991. La tendance générale, avec des degrés divers de précision, est donc d'occulter toute influence étrangère. Ainsi la Lettonie a réalisé cet exploit d'affirmer que seuls des Lettons auraient contribué à l'édification de leur État, ignorant non seulement la Russie d'avant 1917 mais aussi les influences allemande ou suédoise, l'URSS n'étant mentionnée que sur les déportations au Goulag ${ }^{12}$. Cette réécriture de l'histoire va même, on l'a

7 Vadim Smirnov, "Vnechnjaja politika stran pribaltiki v otnochenija Rossiii " ( La politique étrangère des États baltes envers la Russie »), Sovremennaja Evropa, 5, septembre-octobre 2016, p. 44-54. L'auteur est chargé de recherches à l'université de Moscou (MGU), et la revue est publiée par l'Institut d'Europe de l'Académie des sciences de Russie.

8 Ibid., p. 45.

9 Ibid., p. 48 et 50 .

10 Ibid., p. 53.

11 Alexandre Smolar, "Les aventures de la décommunisation ", Critique internationale, 5, 1999, p. $155-166$.

12 Une visite au Musée national d'histoire de la Lettonie, à Riga, est de ce point de vue très instructive ! 
vu, jusqu'à la revendication de l'héritage des légions lettonnes ou estoniennes intégrées dans la Waffen SS et qui sont régulièrement commémorées suscitant à chaque fois une légitime colère de la Russie ${ }^{13}$. Il en est de même, chaque fois qu'il est procédé à la destruction de monuments de l'époque soviétique ou à l'érection d'autres, rappelant la seconde guerre mondiale du côté allemand. Ainsi, dans un cas similaire en 2007, la Russie a-t-elle procédé à des mesures de représailles en piratant les systèmes informatiques de l'Estonie, l'un des pays les plus connectés au monde. Quant aux commémorations de la fin de la seconde guerre mondiale, elles ont fait aussi l'objet de contentieux. Cela a été particulièrement manifeste en 2015. En un mot, il s'agit d'effacer l'histoire de la période soviétique et de relativiser celle de l'époque hitlérienne.

On peut penser que ce qui constitue de toute évidence autant de provocations s'inscrit dans une logique de manœuvre de crise et qu'elle est instrumentalisée de part et d'autre pour servir d'autres desseins. Certes il existe des commissions mixtes d'historiens, notamment entre la Russie et la Lettonie, mais leurs résultats ont été pour le moins modestes quand elles n'ont pas été purement et simplement dissoutes ${ }^{14}$. Si de telles commissions existent avec d'autres pays, telle la Pologne, et si elles effectuent un réel travail d'historien, il reste peu probable, au moins à court terme, que celles-ci débouchent sur la rédaction d'un manuel d'histoire en commun. Autrement dit, l'histoire reste bien une pomme de discorde correspondant au mode conflictuel des relations des petits pays avec plus grand qu'eux, comme on l'a vu précédemment.

L'autre aspect tenant, d'une certaine manière, à l'histoire et qui caractérise les relations de la Russie avec les anciennes Républiques soviétiques, et tout particulièrement avec ses voisins baltes, concerne la situation des minorités russes résidant dans son "étranger proche $»^{15}$.

À l'époque soviétique, nombre de Russes s'étaient installés - volontairement ou non - dans les diverses Républiques composant l'URSS, constituant autant de minorités d'importance variable. Ainsi, à la veille de la disparition de l'URSS, les Russes ne représentaient que $9 \%$ de la population lituanienne mais $28 \%$ en

13 "Au centre de Riga, un cortège à la mémoire des légionnaires lettons de la Waffen SS », Nezavisimaja Gazeta, 17 mars 2017.

14 La commission russo-lettone était présidée, pour la partie russe, par l'académicien Alexandre Tchoubarian et par A. Sunda pour la partie lettone. Elle a suspendu ses travaux le 12 septembre 2014 - fiche pays Lettonie du ministère russe des Affaires étrangères, [http://www.mid.ru/ru/maps/ lv/?currentpage=main-countryla] (consulté le 15 septembre 2017 ).

15 Si le terme "étranger proche " a disparu du vocabulaire diplomatique russe depuis le milieu de la décennie 1990, l'idée est toujours présente. La Russie a toutefois volontairement laissé planer une incertitude concernant l'appartenance des États baltes à l'espace couvert par cette doctrine : CEI ou ex-URSS ? 
Estonie et $33 \%$ en Lettonie ${ }^{16}$. Même si ces chiffres avaient légèrement diminué vingt-cinq ans plus tard, le rejet des populations russes n'en reste pas moins plus fort que dans n'importe quelle autre République ex-soviétique. C'est d'ailleurs dans ces seules Républiques - à l'exception de la Lituanie - qu'a été instauré ce statut, à la limite de la légalité internationale, de "non-citoyens », contribuant ainsi largement au contentieux avec Moscou ${ }^{17}$.

La Russie saura d'ailleurs fort bien instrumentaliser cette situation notamment dans le cadre du Conseil de l'Europe, avant comme après son adhésion à l'organisation. La question du respect des droits de l'homme dans les États baltes a en effet constitué une thématique récurrente du discours russe depuis 1992 et le Conseil de l'Europe a représenté l'une de ses tribunes favorites, surtout après son adhésion en 1996, et ce jusqu'à la crise ukrainienne de 2014.

Avant son admission au Conseil, Moscou avait plus d'une fois protesté contre l'adhésion des trois Baltes et notamment celle de l'Estonie et de la Lettonie, considérant que la violation systématique des droits des minorités (en l'occurrence des citoyens d'origine russe) était incompatible avec l'adhésion de ces pays. Moscou, alors, n'hésitait pas à qualifier la politique menée dans ces deux pays $d^{\prime}$ " apartheid » ou encore d' " épuration ethnique douce ${ }^{18}$. Une fois acquise son adhésion en février 1996 - alors même que la première campagne militaire en Tchétchénie (décembre 1994-août 1996) était encore en cours -, Moscou utilisera régulièrement la tribune du Conseil de l'Europe pour reprendre ses attaques contre le traitement de ces minorités par Tallinn et Riga.

Ces critiques procèdent de la doctrine russe de l'étranger proche, car, même si les Baltes ne sont pas nécessairement inclus dans la zone d'application de la doctrine, l'un de ses principes consiste bien à protéger les populations russes ou russophones où qu'elles se trouvent. Ce principe contribuera naturellement à alimenter les craintes d'une ingérence, voire d'une intervention russe dans les États baltes, crainte qui s'accentuera encore au lendemain de l'annexion de la Crimée par la Russie en 2014.

Pour rester dans le domaine des minorités et des origines des populations de ces pays, Moscou a aussi largement instrumentalisé l'origine géographique de certains des nouveaux responsables politiques et militaires. Il est vrai que, au

16 Ejegodnik BSE 1988 (Annuaire de la grande encyclopédie soviétique), Moscou, Sovetskaja Enciklopedija, 1988, p. 128, 133, 186 (l'édition de 1989 n'indique aucun chiffre).

17 Même si le nombre de ces non-citoyens a fortement diminué depuis 1995, notamment pour des raisons démographiques, ce statut privant de nationalité et de nombreux droits ceux qui ne connaissent pas les institutions et, surtout, qui ne maîtrisent pas la langue du pays reste une épine dans les relations avec la Russie.

18 Jean-Christophe Romer, "L'URSS, la Russie et le Conseil de l'Europe : une relation complexe ", in Jalons pour une histoire du Conseil de l'Europe, M.-T. Bitsch (dir.), Berne, Peter Lang, 1997, p. 214. 
moment de l'indépendance, il n'était pas aisé de trouver un personnel politique compétent en nombre suffisant pour tenir les rênes du pouvoir et qui ne soit pas un produit de l'ancien régime. Certes, il existait quelques dirigeants anciennement membres du parti communiste et qui étaient, comme en Europe centrale, largement "social-démocratisés " au moment de la chute de l'URSS, tel Algirdas Brazauskas, premier secrétaire du PC lituanien avant d'être élu président de la République (1992-1998) puis Premier ministre (2001-2006). Mais il s’agit là plutôt d'une exception. Les nouveaux États indépendants ont donc dû engager un "processus de ré-émigration ${ }^{19}$, c'est-à-dire importer du personnel politique originaire de ces pays et qui, pour la plupart, vivait en Amérique du Nord. Pour ne mentionner que des chefs d'État, on constate que les trois pays ont été concernés : Vaira Vike-Freiberga, présidente de la Lettonie de 1999 à 2007, est venue du Canada ; Valdas Adamkus, président lituanien de 1998 à 2003 puis de 2004 à 2009, était exilé aux États-Unis ; quant à Toomas Endriks Ilves, président de l'Estonie de 2006 à 2016, il venait également des États-Unis, mais il est aussi le seul des trois présidents à ne pas être né dans son pays d'origine. Ce "processus de ré-émigration " n’a pas concerné que la seule classe politique. Les domaines de la défense et de l'économie ont également été concernés, conduisant, selon Moscou, à renforcer les liens de ces pays avec l'Europe du Nord et surtout avec les ÉtatsUnis, et à contribuer non seulement au développement économique rapide de ces pays mais aussi à l'acquisition de logiques transatlantiques. Tout cela n'a, bien entendu, pas manqué d'être relevé comme autant de critiques par la Russie et a engendré une certaine méfiance quant aux intérêts que servaient ces nouveaux responsables, sans pour autant en faire l'objet d'une crise, voire d'un casus belli ${ }^{20}$.

\section{LA SÉCURITÉ AUX FRONTIÈRES}

Le sujet d'affrontement principal et le contentieux le plus lourd dans les relations entre Moscou et ses voisins baltes porte naturellement sur les questions de sécurité. Celles-ci ont largement déterminé tous les autres aspects de ces relations, tant politiques qu'économiques. Cet aspect apparaît dès avant la chute de l'URSS, au moment des négociations dans le cadre de la CSCE du traité sur les forces classiques en Europe (FCE). Le traité a été signé le 19 novembre 1990 à Paris qui, en cette occasion, avait convié des représentants des trois États baltes - encore soviétiques - en tant qu' invités d'honneur ». Ces derniers ont toutefois été expulsés de la salle par la présidence française, à la demande de l'URSS.

Après la confirmation de l'indépendance des trois États baltes, le 6 septembre 1991 et leur adhésion à l'organisation le 14 octobre suivant, la question

19 Vladimir Olentchenko, «Strany Baltii : laboratorija metodov vnechnego upravelnija » (« Les États baltes : laboratoire des méthodes de gestion de la politique étrangère »), Mejdunarodanja Jizn', no 10, 2015.

20 Ibid. 
de leur participation au traité a été abordée le 12 novembre 1991, entre les représentants permanents, auprès de la CSCE, de l'URSS et des États-Unis, qui sont convenus que les trois États ne seraient pas partie au traitée ${ }^{21}$. Ce qui a été confirmé six mois plus tard, le 15 mai 1992, à Tachkent, lors de la répartition des plafonds des armes classiques concernées par les limitations du traité entre les nouveaux États indépendants issus de l'URSS. Ce régime particulier ne manquera pas de susciter des controverses, notamment lors des négociations sur l'adaptation du traité (1999), puis après leur adhésion à l'Alliance atlantique (2004).

Toutefois cette situation a largement perdu de son acuité compte tenu du fait que le traité FCE n'est plus à l'ordre du jour, d'une part parce que son adaptation de 1999 n'a été ratifiée par aucune des puissances occidentales, d'autre part parce que la Russie a suspendu sa participation au traité en 2007 et s'en est retirée de fait en 2015. Certes, la Russie ne peut plus guère contester l'absence des Baltes du traité de 1990 mais, dans son imaginaire, elle aurait sans doute souhaité que ses trois anciennes Républiques devinssent des États neutres à l'instar de la Suède ou, surtout, de la Finlande. Or, c'est bien le contraire qui est en train de se produire puisque, avec l'aggravation des tensions dans la région, c'est la perspective d'une adhésion de la Suède et/ou de la Finlande à l'OTAN qui s'ouvre : ce qui, bien naturellement, suscite de vaines protestations de la part de Moscou. Il est vrai que le comportement souvent agressif de la Russie - la crise ukrainienne n'en est qu'une illustration, à ajouter aux agissements à la limite de la provocation de l'armée russe - ne fait qu'alimenter régulièrement ces tendances chez les neutres. On peut d'ailleurs se demander si la Russie ne ferait pas tout pour pousser les deux pays neutres de la région dans les bras de l'OTAN, permettant à Moscou de légitimer son complexe de citadelle assiégée.

La Suède est aussi créditée d'être, avec la Pologne, à l'origine, en 2008, du projet de " partenariat oriental " de l'UE ${ }^{22}$ qui est perçu par la Russie comme " un outil visant à détacher les États de la CEI [de la Russie] pour les associer à l'UE $»^{23}$. Mais au-delà de ces prémisses, le principal enjeu de sécurité pour la Russie se rapporte d'abord aux représentations traditionnelles de son propre espace. On l'a vu plus haut, la masse continentale russe et les vicissitudes de son histoire ont généré, depuis des siècles, chez les dirigeants comme dans la population, un complexe d'encerclement, c'est-à-dire le sentiment d'être entouré de voisins potentiellement hostiles. Que certains, à l'ouest, dénient à la Russie de

21 Matthieu Chillaud, «Incertitudes stratégiques en Europe septentrionale : les États baltes, l'OTAN et le traité FCE ", Annuaire français de relations internationales, 2002, p. 581.

22 C'est effectivement sur une initiative des ministres des Affaires étrangères des deux pays, Carl Bildt et Radoslaw Sikorski, en mai 2008, qu'est né le Partenariat oriental (PO) officiellement lancé par l'UE un an plus tard, le 7 mai 2009, à Prague.

23 Vladimir Olentchenko, "Rossija i strany Baltii : kontury koncepcii dvuhstoronnyh otnochenij " ("La Russie et les États baltes : contours d'une conception de relations bilatérales »), Mejdunarodanja Jizn', no 9, 2016. 
développer ce sentiment est une chose, le fait est qu'il y existe bien et qu'il y est fortement ancré24. D'où cette volonté de Staline - parmi d'autres motivations de constituer à ses frontières un glacis protecteur fait de pays qui ne "soient pas hostiles à l'URSS ${ }^{25}$. Or, pour nombre de Russes, ce glacis a été perdu en 1989 et ce, sans que le Kremlin réagisse. D'où ce sentiment que les dizaines de millions de morts soviétiques de la " grande guerre patriotique » auraient été sacrifiés pour $\operatorname{rien}^{26}$. Un quart de siècle plus tard, cet argument a quelque peu disparu mais n'en reste pas moins prêt à ressurgir à la moindre occasion, comme lors du lancement du processus d'élargissement de l'Alliance atlantique.

\section{LES ÉLARGISSEMENTS DE L'OTAN}

La première vague, qui concernait les trois pays d'Europe centrale (Hongrie, Pologne, République tchèque) a, de surcroît, également été perçue comme un retournement d'alliance, démonstration que l'OTAN restait cette alliance de guerre froide résolument orientée contre l'URSS d'hier et la Russie aujourd'hui. Certes, le siège de Bruxelles ne manque jamais de chercher à rassurer les Russes par des paroles lénifiantes ${ }^{27}$ en affirmant que l'Alliance n'est " dirigée contre personne ". Ce n'est évidemment pas la conception des pays qui ont adhéré à l'Alliance tant lors de la première que de la deuxième vague des élargissements et notamment de la Pologne et des États baltes.

Les négociations sur le premier élargissement se déroulent en 1996-1997 et sont menées par Evgeni Primakov alors ministre des Affaires étrangères, du côté russe, et Warren Christopher puis Madeleine Albright du côté des États-Unis. Primakov, excellent diplomate, a incontestablement réussi à négocier au mieux des intérêts d'un pays qui n'était alors pas en position de force. L' « Acte fondateur sur les relations, la coopération et la sécurité mutuelles entre l'OTAN et la Fédération de Russie » a représenté pour Moscou l'optimum de ce qu'elle pouvait espérer obtenir. Mais Primakov a aussi indiqué à ses interlocuteurs les limites à ne pas dépasser : « il y a deux lignes rouges à ne pas dépasser ». La première concerne le refus d'un "rapprochement des infrastructures militaires de l'OTAN des frontières de la Fédération de Russie ", un thème récurrent du discours diplomatico-stratégique russe sur lequel on reviendra. La seconde est le refus russe d'une "participation à l'OTAN des États baltes, ni d'aucune ancienne République de l'Union soviétique $»^{28}$.

24 Voir par exemple Le Monde, 31 mars 2017.

25 Pour reprendre les termes d'un message adressé par Roosevelt à Staline lors de la conférence de Yalta et qui concernait la Pologne.

26 La propagande soviétique parlait de 20 millions. Les historiens russes ont revu depuis 1991 ce chiffre à la hausse atteignant jusqu’à 27 millions.

27 Voir par exemple le discours de Jens Stoltenberg, secrétaire général de l'OTAN, à la conférence annuelle sur la sécurité, Munich, le 18 février 2017, [http://www.nato.int/cps/en/natohq/ opinions_141632.htm?selectedLocale=en] (consulté le 22 février 2017) .

28 Evgenii Primakov, Au cour du pouvoir, Paris, Éditions des Syrtes, 2002, p. 178. 
Dans ces conditions, comment la Russie a-t-elle pu accepter que la deuxième vague d'élargissement inclue justement les trois États baltes ? Il faut se replacer dans les conditions de l'arrivée au pouvoir de Vladimir Poutine en 2000 et de la ligne directrice de son premier mandat.

La situation internationale a effectivement profondément changé entre les deux premières vagues d'élargissement de l'Alliance avec notamment la deuxième campagne militaire en Tchétchénie et l'accession au pouvoir de Vladimir Poutine, pour la Russie, et, pour les États-Unis, l'élection de George W. Bush et les attentats du 11 septembre 2001. Le premier mandat de Vladimir Poutine (2000-2004), destiné à remettre de l'ordre après la confusion eltsinienne, a été marqué par une orientation résolument européenne mais aussi par des convergences d'intérêts entre Moscou et Washington après septembre 2001 : Poutine a besoin de Bush pour légitimer ses actions en Tchétchénie et inversement les États-Unis ont besoin de la Russie - et de l'Asie centrale ex-soviétique - comme base arrière pour leurs opérations en Afghanistan. Dès lors, Washington impose à Moscou d'avaler un certain nombre de couleuvres, l'élargissement décidé au sommet de Prague en novembre 2002 étant une de celles-là.

Même si cet élargissement, pour la première fois, empiétait sur le territoire de l'ancien Empire, les réactions de la Russie n'ont été que purement symboliques. La seule concession notable - pour autant que l'on puisse parler de concession a été la transformation du Conseil conjoint permanent OTAN-Russie (CCP) en Conseil OTAN-Russie (COR/NRC) lors du sommet OTAN-Russie qui se tient à Rome le 28 mai 2002. Le COR est ainsi conçu comme devant marquer une "nouvelle qualité des relations... entre partenaires égaux aux intérêts communs $»^{29}$.

Mais ce rapprochement est de courte durée puisque l'on retrouve une nouvelle source de contentieux avec l'intervention, aussi illégale qu'illégitime, des ÉtatsUnis en Irak qui suscite le soutien de huit pays membres de l'Alliance dont les trois nouveaux adhérents. Cette "Lettre des Huit " a été suivie par une "Lettre des Dix " pays du " groupe de Vilnius ", c'est-à-dire des candidats à l'adhésion à l'Alliance participant au MAP (Membership Action Plan) dont les trois Baltes. L'alignement inconditionnel de ces pays sur les positions de Washington n'a naturellement pas amélioré les relations de la Russie avec ses voisins, baltes notamment ${ }^{30}$.

29 Déclaration des chefs d'État et de gouvernement des États membres de l'OTAN et de la Fédération de Russie, Rome, 28 mai 2002, [http://www.nato.int/nrc-website/media/69558/2002.05.28_nrc_ official_statement_hos.pdf] (consulté le 15 septembre 2017).

30 Lettre des huit pays d'Europe pour un front uni face à l'Irak (Lettre des Huit), 30 janvier 2003, [http://archive2.grip.org/bdg/g2026.html] (consulté le 15 septembre 2017) et Déclaration des pays du groupe de Vilnius (Lettre des Dix), 5 février 2003, [https://www.diploweb.com/ue/crise2003.htm] (consulté le 15 septembre 2017). 


\section{GESTICULATIONS DANS L'ORDRE MILITAIRE}

Mais le contentieux le plus grave et le plus durable apparait peu après, en 2007, avec la décision de Washington - qui avait annoncé, en décembre 2001, son retrait du traité SALT1-ABM de 1972 qui devient effectif six mois plus tard - de déployer des systèmes de défense antimissile balistique (DAMB) en Pologne et, dans un premier temps, en République tchèque puis en Roumanie. Dès lors, les Baltes seront souvent associés par Moscou à la Pologne, car, dans la polémique sur la DAMB, qui ira crescendo, ils ressortissent à la même logique en termes de sécurité régionale. L'argument avancé par la Russie consiste à affirmer que si les États-Unis veulent déployer ces systèmes dans la partie orientale du Vieux Continent, ce n'est pas pour contrer une éventuelle attaque balistique provenant d'Iran, comme ils le proclament, mais bien aussi contre la Russie ${ }^{31}$. Ce qui n'est qu'à demi faux. À demi, car ce n'est pas la dizaine de missiles antimissile déployés en Pologne qui menaceront les systèmes stratégiques centraux de la Russie. Par contre, les systèmes de détection qui devaient être déployés en République tchèque puis en Roumanie peuvent très bien être conçus aussi pour permettre une extension de leur capacité et faire office de système de détection avancée des missiles russes. Dans ce domaine, le discours - la propagande - prime souvent sur la réalité, d'un côté comme de l'autre.

Mais il est aussi évident que, en termes de perception de la menace et de la sécurité, la crise ukrainienne a fait office de catalyseur des craintes des États baltes qui disposaient là d'un argument facile pour laisser penser qu'ils pourraient constituer la prochaine étape d'une volonté russe de reconquête impériale. Cet argument leur a permis d'obtenir, sans difficulté, de Bruxelles et de Washington des mesures de "réassurance ».

Se sont ainsi succédé des vols menaçants de l'OTAN dans la région baltique ${ }^{32}$ répondant à des vols tout aussi provocateurs de l'aviation russe dans la même région et au-delà. À moins que ce ne soit le contraire : Bruxelles et Washington, d'un côté, Moscou, de l'autre, s'accusent mutuellement de provocations dignes de la "guerre froide ", à mesure de ces incursions aux limites des frontières de l'espace aérien et maritimes de l'autre ${ }^{33}$. À cela s'ajoutent des exercices militaires

31 Selon Moscou, cet argument ne devrait plus pouvoir être mis en avant depuis la signature de l'accord sur le nucléaire avec l'Iran, le 14 juillet 2015.

32 Voir notamment la conférence de Sergeï Choïgu au MGIMO, reproduite dans Krasnaja Zvezda, 22 février 2017.

33 Comme du temps de la guerre froide, on constate toujours des incursions de sous-marins russes à proximité des côtes de la Norvège ou de la Suède, suscitant de tels fantasmes que, dans quelques cas, des bancs de poissons ont pu être confondus avec des sous-marins. 
de plus ou moins grande ampleur, organisés également à proximité des frontières de l'autre. La presse russe se gaussera d'ailleurs d'un discours occidental sur la " menace russe » et sur la volonté de constituer un «Schengen militaire » ${ }^{34}$.

De leur côté, les pays de l'OTAN dénoncent régulièrement les manœuvres russes qui, sous ce prétexte, s'approchent parfois de trop près de bâtiments ou d'installations de l'OTAN. Quant aux exercices militaires organisés à Kaliningrad - qui redevient un bastion avancé de la défense russe -, ils ont régulièrement constitué le prétexte pour mettre en œuvre une "diplomatie de l'Iskander », pendant régional de la "diplomatie du S $300 »^{35}$. Cette " diplomatie " consiste à annoncer le déploiement de ces missiles tactiques «Iskander ", d'une portée de 4 à 500 kilomètres, dans l'enclave de Kaliningrad et donc susceptibles d'atteindre les principales capitales d'Europe du Centre et du Nord - Berlin, Varsovie, Copenhague ou Stockholm. Elle a dans un premier temps été utilisée sous les prétextes d'abord des élargissements de l'OTAN puis du déploiement des systèmes DAMB. Cette stratégie déclaratoire ne s'était guère concrétisée, sauf, temporairement, à l'occasion de manœuvres dans l'enclave. Or, depuis octobre 2016 des batteries d'Iskander y ont bien été installées de façon permanente et hors du cadre d'exercices militaires sous le prétexte de répondre aux mesures de " réassurance " de l'OTAN en Pologne et dans les États baltes : une «mesure nécessaire pour dissuader toute éventuelle agression de l'OTAN dans la région ${ }^{36}$.

Dès lors, on peut se demander s'il n'y aurait pas une corrélation entre cette décision et le changement intervenu dans le discours stratégique russe en décembre 2015 avec, bien sûr, une fois encore, la crise en Ukraine comme toile de fond. En effet, pour la première fois dans les textes programmatiques russes, Moscou considère l'OTAN ou, plus précisément, "le rapprochement des infrastructures militaires de l'alliance des frontières de la Fédération de Russie ", non plus comme un "risque " (littéralement : "danger» : opasnost) mais comme une "menace» (ugroza). Ce changement, présent dans la «stratégie de sécurité nationale ", adoptée le 31 décembre 2015, et la distinction entre les deux catégories - risque/menace - a été peu relevé à l'ouest alors que, pour la Russie, il s'agit d'un véritable changement d'approche $\mathrm{du}$ " bloc » occidental. D'où l'importance de savoir ce que pense et dit l'autre!

34 Notamment à l'occasion des manœuvres de l'OTAN «Anaconda 2016 » au printemps 2016 : Krasnaja Zvezda, 18 mai 2016, 8 juin 2016 ou Nezavisimaja Gazeta, 6 juin 2016.

35 Moscou a plus d'une fois annoncé la livraison de ces missiles sol-air à des pays amis - mais non nécessairement alliés de la Russie : de la Serbie en 1999 à l'Iran.

36 Voir notamment " Desjat naibolee vajnyh sobytij 2016 goda " ("Les dix événements les plus importants de $2016 »)$, Nezavisimoe voennoe obozrenie, 23 décembre 2016. 


\section{ConClusion}

En conclusion, on peut se demander si la Russie constitue une véritable menace militaire pour les Baltes et si l'OTAN et certains de ses pays membres en représentent pour la Russie. Comme souvent, mais pas toujours, la responsabilité de cette montée en puissance de l'hostilité entre les deux ensembles est partagée : agressivité du petit à l'égard du grand et réponse disproportionnée du grand envers le petit, surtout si ce dernier est soutenu par encore plus grand ! Bien sûr, pour Moscou, toute la responsabilité revient à l'OTAN et à ces " petits pays " qui profitent de leur situation pour obliger leurs alliés à les soutenir. Et là encore, comme au temps de l'affrontement bipolaire que certains, d'un côté comme de l'autre, souhaiteraient bien reconstituer, la désinformation des uns sert celle des autres et inversement, y compris dans le domaine de la guerre de l'information électronique et des cyberattaques, sont finalement peu mentionnées par les milieux tant politiques que journalistiques russes mais qui sont aussi bien réelles.

On peut alors se demander si Moscou ne chercherait pas à maintenir une véritable incertitude quant à ses intentions à l'égard de ses anciennes " provinces ", de manière à les laisser dans une situation non pas de conflit mais au moins de "crise gelée » qui constitue autant de variables d'ajustement de la diplomatie russe. À l'inverse, le refus des trois États baltes d'admettre que la Russie est leur grand voisin et qu'ils n'en changeront pas de sitôt - quelque encombrant qu'il puisse être - n'arrange pas la situation. 